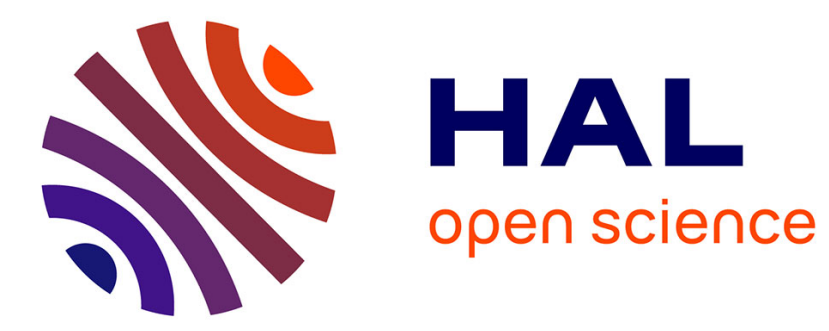

\title{
Dissipative aspects in high cycle fatigue
}

Eric Charkaluk, Andrei Constantinescu

\section{To cite this version:}

Eric Charkaluk, Andrei Constantinescu. Dissipative aspects in high cycle fatigue. Mechanics of Materials, 2009, 41 (5), pp.483-494. 10.1016/j.mechmat.2009.01.018 . hal-00376738

\section{HAL Id: hal-00376738 \\ https://hal.science/hal-00376738}

Submitted on 1 Jun 2021

HAL is a multi-disciplinary open access archive for the deposit and dissemination of scientific research documents, whether they are published or not. The documents may come from teaching and research institutions in France or abroad, or from public or private research centers
L'archive ouverte pluridisciplinaire HAL, est destinée au dépôt et à la diffusion de documents scientifiques de niveau recherche, publiés ou non, émanant des établissements d'enseignement et de recherche français ou étrangers, des laboratoires publics ou privés. 


\title{
Dissipative aspects in high cycle fatigue
}

\author{
Eric Charkaluk ${ }^{\mathrm{a}, *}$, Andrei Constantinescu ${ }^{\mathrm{b}}$ \\ ${ }^{a}$ Laboratoire de Mécanique de Lille - CNRS UMR 8107, boulevard Paul Langevin, 59655 Villeneuve d'Ascq cedex, France \\ ${ }^{\mathrm{b}}$ Laboratoire de Mécanique des Solides - CNRS UMR 7649, École Polytechnique, 91128 Palaiseau cedex, France
}

\begin{abstract}
The fatigue crack initiation phenomenon in metals is essentially associated to the gliding of dislocations and the creation of slip bands which characterize irreversible damaging mechanisms at the grains scale. A multiscale approach is then interesting to establish a link between the scale of the structure submitted to cyclic loading and the scale of damage. Some stress based criteria were previously proposed in this framework based on the shakedown theories, as Dang Van or Papadopoulos ones. The same approach is developed in this paper in the usual Thermodynamics of Irreversible Processes (TIP) framework in order to study the link between dissipation, shakedown and fatigue damage. Some recent results in metals fatigue coming from infrared thermography experiments are then interpreted through the TIP; the proposed framework seems to be particularly relevant to define a more general feature for the study of the fatigue phenomenon.
\end{abstract}

\section{Introduction}

It is now well known that the fatigue phenomenon in metals is essentially associated to the gliding of dislocations and the creation of slip bands which characterize irreversible damaging mechanisms at the grains scale. A review of these mechanisms can be founded for example, in the monograph of Suresh (1998), in the pioneering works of Mughrabi (1983), in the papers of Magnin et al. $(1984 a, b)$ or in the recent synthesis of Lukas and Kunz (2001, 2002, 2004).

The behavior of mono- and polycrystals is dependent of the loading level and other factors such as environment, temperature, .... For a Representative Elementary Volume of material (REV), they can be summarized using the following scheme:

- If the loading level is very low, only a single slip system can be considered in each grain with a shear stress below the resolved yield stress on this slip plane. An elastic response is therefore obtained in all the grains and no slip bands can be observed. The macroscopic response, on the REV boundaries, is then also elastic.

\footnotetext{
* Corresponding author. Tel.: +33 3203371 82; fax: +33 320337153 . E-mail address: eric.charkaluk@univ-lillel.fr (E. Charkaluk).
}

- If the loading level is increased but is still low, a single slip system can be considered in each grain. The shear stress might exceeds resolved yield stress in some grains possessing a properly oriented crystallographic system with respect to the active slip system and the loading direction. In such grains, the mechanisms of dislocations gliding and slip bands creation can be activated. At the grain surface, such bands can be observed. This heterogeneous plastic behavior, depending on the grains orientations, has recently been quantified by Korsunsky et al. (2004) by diffraction measurements. Some observed grains display a quasi elastic response while others cyclically plastify. However, if this plastic behavior is confined in a few grains, the macroscopic response, on the REV boundaries, remains quasi-elastic.

- If the loading level becomes more important, it can induce two effects. On the one hand, the quantity of plastic grains becomes more important Sauzay, 2006 and, on the other hand, multiple slip systems can be activated in the same grain. Plastic behavior is then no more confined, is generalized in all the REV, and the macroscopic behavior becomes elastoplastic.

The three schemes correspond to three different fatigue domains, which can easily be described by a Wöhler's 


\section{Nomenclature}

\begin{tabular}{|c|c|}
\hline $\mathbb{C}, \mathbb{P}$ & $\begin{array}{l}\text { respectively, elastic moduli and Hill's forth rank } \\
\text { tensors }\end{array}$ \\
\hline $\mathbb{\square}, \mathbb{J}, \mathbb{K}$ & forth rank identity tensors \\
\hline$\Sigma, \sigma$ & $\begin{array}{l}\text { macroscopic, respectively, mesoscopic stress } \\
\text { tensors }\end{array}$ \\
\hline $\boldsymbol{E}, \boldsymbol{\varepsilon}$ & $\begin{array}{l}\text { macroscopic, respectively, mesoscopic total } \\
\text { strain tensors }\end{array}$ \\
\hline $\boldsymbol{E}^{p}, \boldsymbol{\varepsilon}^{p}$ & $\begin{array}{l}\text { macroscopic, respectively, mesoscopic plastic } \\
\text { strain tensors }\end{array}$ \\
\hline $\boldsymbol{E}^{e}, \boldsymbol{\varepsilon}^{e}$ & $\begin{array}{l}\text { macroscopic, respectively, mesoscopic elastic } \\
\text { strain tensors }\end{array}$ \\
\hline $\boldsymbol{x}$ & mesoscopic hardening stress tensor \\
\hline $\boldsymbol{I}$ & second rank identity tensor \\
\hline$\rho$ & mesoscopic residual stress tensor \\
\hline$\Phi$ & intrinsic dissipation \\
\hline$d_{p}$ & plastic dissipation \\
\hline$\Delta w_{p}$ & $\begin{array}{l}\infty) \text { plastic dissipated energy, respectively, per } \\
\text { cycle and cumulated }\end{array}$ \\
\hline$\tau, \sigma_{h}$ & $\begin{array}{l}\text { mesoscopic respectively, resolved shear stress } \\
\text { and hydrostatic pressure }\end{array}$ \\
\hline
\end{tabular}

$k \quad$ radius of the hypersphere enclosing the deviatoric stress path

$\alpha_{i}, \beta_{i}$ material dependent HCF and LCF criteria parameters

$\sigma_{D}, \sigma_{D t}, \sigma_{D b}$ endurance limits, specifically in torsion and bending

$\sigma_{y} \quad$ mesoscopic yield stress

$\lambda, \mu \quad$ Lamé's parameters

$E, K, v \quad$ Young's and bulk moduli and Poisson's ratio

$h \quad$ mesoscopic hardening modulus

$a, b$ parameters related to the homogenization

scheme

$f_{v} \quad$ volumic fraction of plastic inclusions

$\kappa, \alpha, C_{v}, \rho$ thermal conductivity and expansion, specific heat and density

$\tau_{e q} \quad$ constant representative of the thermal exchanges

$T$ absolute temperature

$r \quad$ external heat source

$\gamma_{p} \quad$ plastic multiplier

$N, N_{f} \quad$ number of cycles, in particular to failure

curve, schematically represented on the Fig. 1. The first case, presenting an elastic shakedown at all physical scales, can be easily associated to infinite lifetimes and unlimited endurance. The second case, corresponding to a localized cyclic plastic behavior in some well oriented grains, conducts to High Cycle Fatigue (HCF) and a limited endurance. Finally, the last case, associated to a generalized plastic behavior in the REV, conducts to Low Cycle Fatigue (LCF) phenomenon. Historically, in the case of HCF, as the macroscopic behavior of the REV and, as a consequence, that of the complete structure, remains elastic, fatigue criteria were based on the stress tensor. This implies the expressions of Gough and Pollard (1935), Crossland (1956) or Sines and Waisman (1959). In the case of LCF, as the macroscopic behavior of the REV and that of the complete structure can be considered as completely plastic, fatigue criteria were based on the plastic or total strain tensors. This conducts, for example, to the pioneering approach of Manson (1953) and Coffin (1953) or the proposition of Smith et al. (1970). However, as reminded, the fatigue

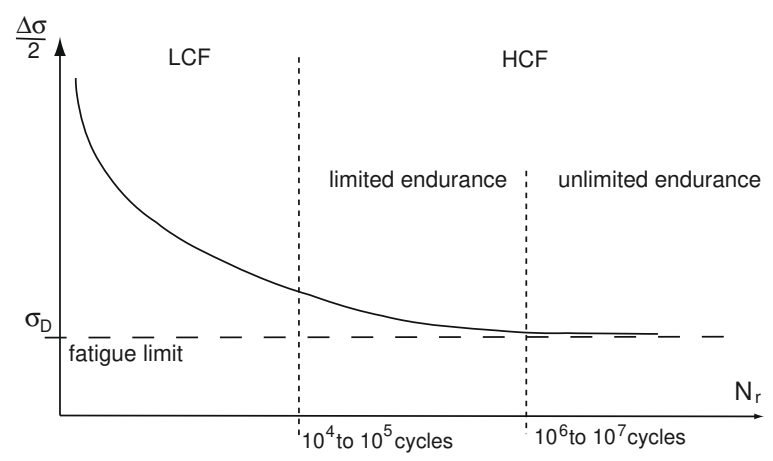

Fig. 1. Schematic representation of the Wöhler's curve defining the different fatigue domains (HCF and LCF).

mechanisms are the same in all the cases, the fatigue domains depending essentially on the spatial expansion of the plasticity. Therefore, these domains can rather be related to different scales: the grain in a REV in HCF and the complete material volume in LCF.

In order to establish a common approach in HCF and LCF, it is proposed to analyze the link between the dissipation generated by the cyclic plastic activity and the crack initiation; this conducts to consider this dissipation as a damage indicator, as proposed a long time ago by Halford (1966). It is therefore proposed here to revisit the historical way, started by Farren and Taylor (1925) by emphasizing the importance of the plastic work during the metal deformation under monotonic loading. These authors measured the mechanical energy dissipated during a quasi-static tensile test, as well as the ratio between the energy stored in the material and the one dissipated by heat production and leading to a temperature variation of the specimen. More recently, Chrysochoos (1985) proposed a complete thermodynamical framework in order to describe this thermomechanical coupling and quantified the evolution of the temperature by infrared thermography. In the case of cyclic loadings, a first major contribution is actually due to Dillon (1962a,b, 1963, 1966) in a series of experiments on the cyclic torsion of copper and aluminum tubes. In Dillon (1963), one of his major observations is that the thermoplastic coupling during a cyclic torsion test induces a positive dissipation which conducts to a constant increase of the temperature, as displayed in Figs. 2 and 3.

At this point, no particular link is drawn between the observations and the fatigue phenomenon. In the fatigue framework, a particularly exhaustive study of Stärk (1980) may be stated, that established correlations between dissipative domains and loading levels for a stainless steel and a gray cast iron in HCF. His observations on cyclic loading can be summarized as follows: 

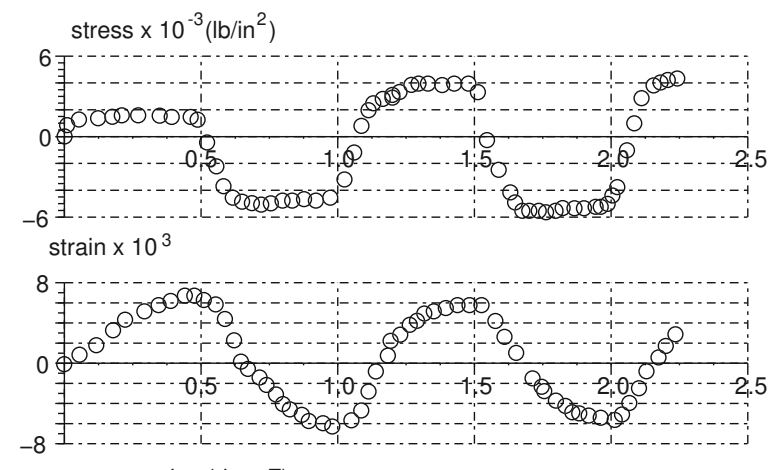

temperature rise (deg. F)

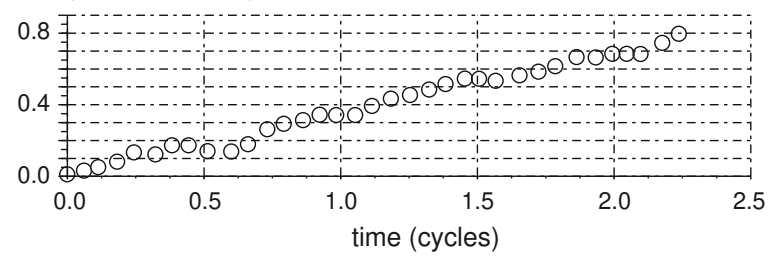

Fig. 2. Evolution of the temperature in the first cycles for an aluminum tube under cyclic torsion (Dillon, 1963).

- An asymptotic temperature state is reached up to a certain loading level, as illustrated on Fig. 4. This is in accordance with the remark made previously by Dillon (1962a,b, 1963, 1966). The fatigue limit of this particular steel is about $310 \mathrm{MPa}$ (Stärk, 1980) and the asymptotic state is observed when the loading is below this limit.

- The asymptotic thermal field in the specimen is loading dependent (see Fig. 5). For low levels of applied loading, the temperature remains close to the initial temperature and almost uniform. This can be explained by localized plasticity in a few spatially spreaded grains, as previously described. The temperature field becomes uniform by heat conduction and the main heat exchanges with the environment are realized by convection. For high level of applied loadings, a parabolic temperature distri-

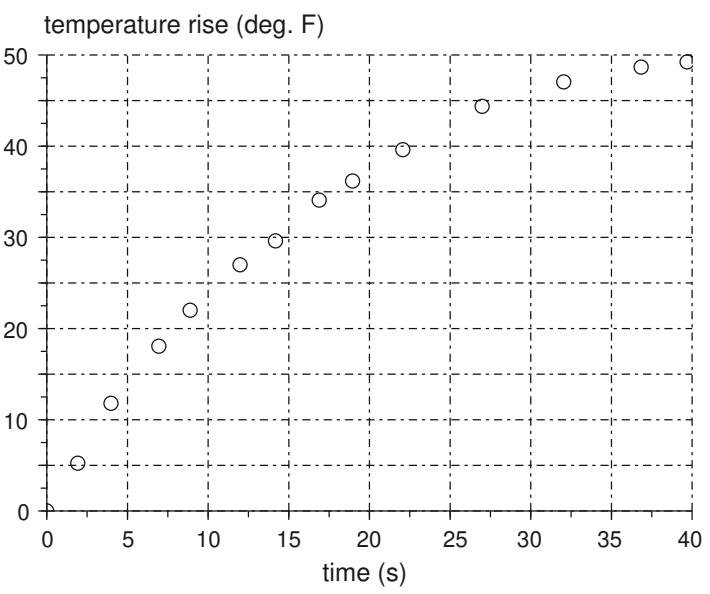

Fig. 3. Evolution of the temperature for an aluminum tube under cyclic torsion (Dillon, 1963).

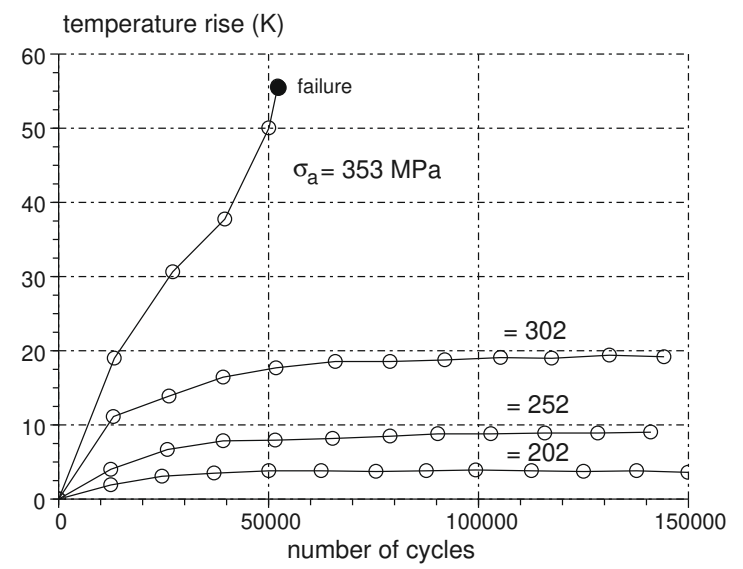

Fig. 4. Evolution of the temperature for a stainless steel (X10CrNiMoTi 18 10) specimen under reversed tension. The fatigue limit of the steel considered here is about $310 \mathrm{MPa}$ (Stärk, 1980).

bution is observed in the specimen. In this case, the plasticity is more extended spatially and creates an almost uniform heat source. The main exchanges with the environment are then realized through convection and the grip systems of specimen. This difference also explains the evolutions observed on Fig. 4: below the fatigue limit, in a few cycle, a thermal equilibrium is obtained between the heat sources and the thermal environment, which is not the case as soon as the loading exceeds the fatigue limit.

Stärk's analysis presents a first direct link between fatigue limit and thermal dissipation. However, the first extended discussion of the phenomena within a thermodynamical framework was certainly proposed by Luong $(1995,1998)$ and enables the determination of the fatigue limit by using infrared thermography technique, as illustrated in Fig. 6 coming from Luong (1998). This result is obtained for an XC55 steel specimen in HCF and shows that

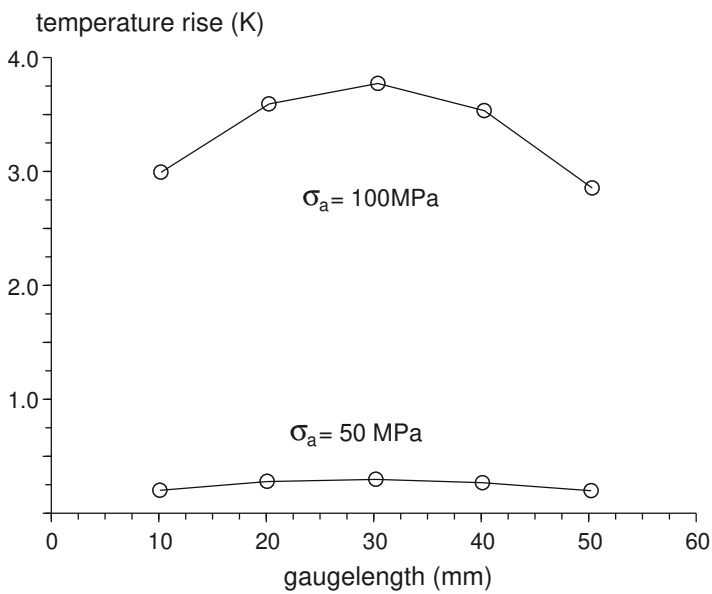

Fig. 5. Thermal gradients along the specimen length for a reversed tension test under different stress amplitude (Stärk, 1980). The material is a stainless steel (X10CrNiMoTi 18 10). 


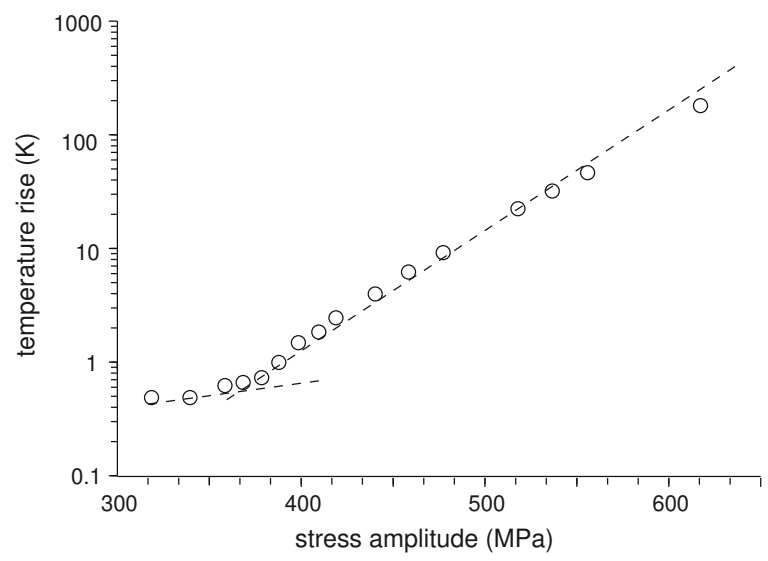

Fig. 6. Results obtained by Luong (1995, 1998), showing the change in dissipative regimes before and after the fatigue limit for a XC55 steel specimen under rotative bending. The fatigue limit is equal to $399 \mathrm{MPa}$ and is estimated by using a Staircase method.

the change in the dissipative behavior corresponds to the transition between unlimited and limited endurance in the fatigue life. The fatigue limit is equal to $399 \mathrm{MPa}$ and is estimated by using a Staircase method. For Luong, in a $\left(\log (\Delta T), \sigma_{a}\right)$ diagram, it corresponds approximately to the intersection between both dotted lines on Fig. 6 . Following this paper, numerous studies were realized to verify this relation on different materials: Fargione et al. (2002) tested different steels and Krapez and Pacou (2002), several aluminum alloys and steels. Recently, Liaw's team withdraw the Luong's framework to the study of many aeronautical alloys (Jiang et al., 2001a,b, 2004) in HCF and LCF. Their observations are totally in accordance with Stärk's one (Stärk, 1980) (thermal gradients, cyclic thermal behavior,...). A comparison of such analysis can be realized, based for example on Luong (1998) and Cugy and Galtier (2002) results on three different steels. This comparison is presented on Fig. 7 , in a $\left(\Delta T, \sigma_{a}\right)$ diagram. The stress range has been divided here by the corresponding fatigue limit (399 MPa for the XC55 steel, $235 \mathrm{MPa}$ for

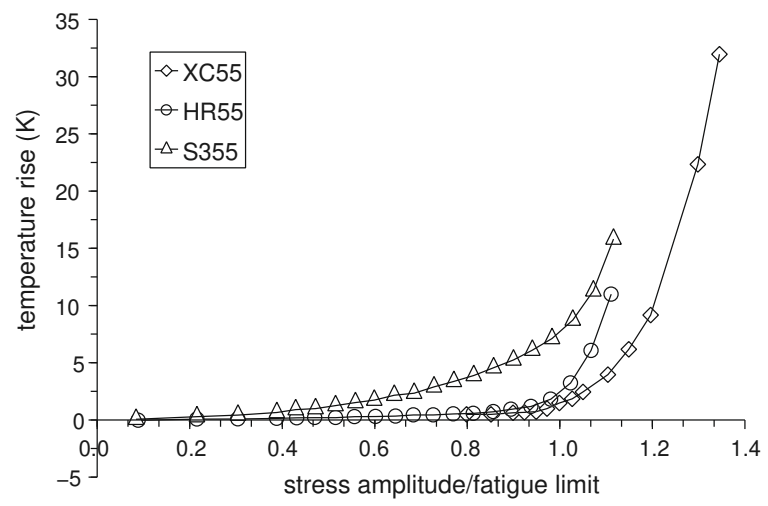

Fig. 7. Results obtained by Luong $(1995,1998)$ on a XC55 steel and by Cugy and Galtier (2002) on HR55 and S355 steels. The stress amplitude is divided by the corresponding fatigue limit: 399 MPa for the XC55 steel, 235 MPa for the HR55 and S355 steels. the HR55 and S355 steels). It exhibits different thermal behaviors: S355 dissipated heat below the fatigue limit which is quite not the case for XC55 and HR55. Cugy and Galtier (2002) underline that the microstructures of S355 and HR55 are totally different: S355 is made of ferrite for $94 \%$ and pearlite for $6 \%$ as HR55 is made of ferrite for $40 \%$ and bainite for $60 \%$. Therefore, microstructure seems to have an important effect on the dissipative behavior below and above the fatigue limit, even if no further discussion on this particular point was pointed out in the literature. A more formal discussion associating fatigue and temperature variations can be founded in the recent works of Boulanger (2004) and Doudard (2004). The discussed papers show the link between the fatigue phenomenon, whether in HCF or LCF and the mechanical dissipation. Moreover, this link seems to be cast within an elastoplastic thermodynamical framework.

The objective of this work is to make another step toward the construction of a unified framework in fatigue based on energy dissipation and the present work will focus on HCF. In this article, the link between elastic shakedown, fatigue limit and dissipated energy is established, based on a brief description of the Dang Van's multiscale approach in HCF. Next, the coupled heat equation is presented, as derived in the framework of the continuum thermodynamics at the mesoscopic scale and established for different self-consistent homogenization schemes. Then, a numerical simulation of the temperature evolution, under cyclic loading, at the macroscopic scale is compared to recent experimental results of Doudard et al. (2005) and Boulanger et al. (2004) and the validity of the homogenization schemes is discussed. The HCF mesoscopic approach enables an extension of the concepts to LCF fatigue which will further be addressed.

\section{A dissipative framework in fatigue}

\subsection{Assumptions of the Dang Van and Papadopoulos HCF model}

A straightforward link between mechanical dissipation and fatigue damage stems from the high cycle metal fatigue theory of Orowan (1939), and the followers model of Dang Van (1973) and Papadopoulos (1987). Starting from the observation that the cyclic evolution of isolated grains in polycrystals submitted to complex loadings can thereby be resumed by the creation of localized slip bands, a cyclic plastic activity in slip bands and the nucleation of microcracks until the creation of a macrocrack, Dang Van (1973) and Papadopoulos (1987) based their models on the next framework of assumptions: (i) the fatigue damage is controlled by mechanisms at the grain scale and therefore a description at this mesoscopic scale is necessary; (ii) at this scale, most of the metallic materials are aggregates of cubic crystals with a random distributed crystallographic orientations, which can be considered isotropic and homogeneous at the macroscopic scale; (iii) among all grains and possible slip planes, only some well oriented slip planes, maximizing the shear stress for a given loading path, will develop plasticity and create localized slip bands inducing crack initiation; (iv) below the fatigue limit, 
microscopic plastic strains homogenize to negligible macroscopic plastic strains, which matches the fact that macroscopic stresses are small with respect to the yield limit; (v) mesoscopic plasticity is determined by isotropic and kinematic hardening rules and the evolution of the mesoscopic yield limit $\tau_{s}$ will initially evolves and then saturates under an imposed cyclic strain loading, as illustrated on Fig. 8. This evolution is inspired by the results of Winter (1974) who associates the saturation with the creation of Persistent Slip Bands (PSB). This idea is then implicitly generalized by Dang Van (1973) and Papadopoulos (1987) to many other materials which do not exhibits such particular three-dimensional dislocation structures, but in which development of slip bands of any kind is observed under cyclic loading. As a consequence, the natural framework of the multiscale approach proposed by Dang Van et al. (Dang Van, 1973; Papadopoulos, 1987), is a representative elementary volume (REV) with an elastic macroscopic behavior and some plasticity localized only in some grains. The simplest mesoscopic model is a plastic Eshelby inclusion in an elastic matrix. As described before, the behavior of the plastic inclusion can be resumed to a two stage evolution of its yield limit (see Fig. 8). Then, the macro- and mesoscopic stress tensors denoted by $\Sigma$ and $\sigma$, as well as the macro- and mesoscopic strain tensors denoted by $\boldsymbol{E}$ and $\boldsymbol{\varepsilon}$ are linked using a homogenization scheme (Bornert et al., 2001a). One possible and well adapted is the SelfConsistent (SC) scheme of Kröner, detailed in Appendix A. The unlimited endurance condition, i.e. conducting to infinite lifetimes, corresponds then, in this framework (Dang Van, 1973; Papadopoulos, 1987), to an elastic shakedown state in the plastic inclusion. However, since the works of Dang Van (1973) and Papadopoulos (1987), many investigations were realized on the link between crack initiation and the concept of fatigue limit: the endurance limit is rather close to the stress amplitude below which a short crack initiated in an individual grain is unable to propagate toward the neighboring grains. Then, the definition of the endurance limit proposed by Dang Van (1973) and Papadopoulos (1987), corresponding to the non initiation of cracks in individual grains, is much more a lower bound of the real endurance limit of the material.

The Dang Van and the Papadopoulos criteria are both based on a Lin-Taylor homogenization assumption (Lin,

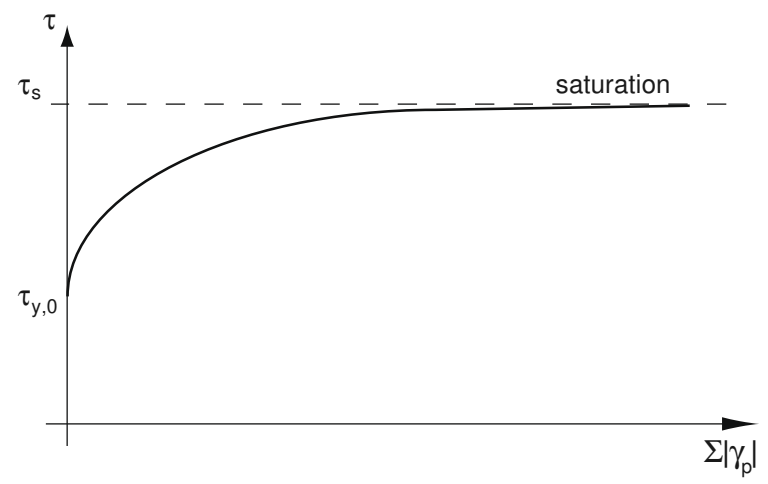

Fig. 8. Schematic hardening curve of a monocrystal under cyclic loading. $\tau$ is the resolved shear stress on the slip plane.
1957; Taylor, 1938). The Dang Van criterion is defined in terms of the hydrostatic pressure, $\sigma_{h}$, and of the mesoscopic resolved shear stress $\tau$. The Papadopoulos criterion depends also on $p$ and on the radius $k$ of the smallest hypersphere enclosing the deviatoric stress path. In this last case, the crack nucleation condition is deduced from the shakedown theorem of Mandel et al. (1977). These criteria can be written as:

$$
\begin{aligned}
& \max _{\boldsymbol{n}, t}\left[\left\|\tau(\boldsymbol{n}, t)-\tau_{m}(\boldsymbol{n})\right\|+\alpha_{d v} \sigma_{h}(t)\right] \leqslant \beta_{d v} \quad \text { Dang Van } \\
& \max _{t}\left(k(t)+\alpha_{p a} \sigma_{h}(t)\right) \leqslant \beta_{p a} \quad \text { Papadopoulos }
\end{aligned}
$$

The material parameters $\alpha_{d v}, \alpha_{p a}, \beta_{d v}$ and $\beta_{p a}$ are defined in terms of the endurance limits in reversal torsion tests $\sigma_{D t}$ and bending experiments $\sigma_{D b} . \boldsymbol{n}$ is the normal to the shear plane. $\tau_{m}(\boldsymbol{n})$ is the vector which points to the center of the smallest circle circumscribing the path described by the tip of the shear stress vector $\tau(\boldsymbol{n}, t)$ on the plane defined by the unit normal vector $\boldsymbol{n}$. In the Eq. (2), $k(t)$ is defined as:

$k(t)=\sqrt{\frac{3}{2}\left(\boldsymbol{s}(t)-\boldsymbol{s}_{m}\right):\left(\boldsymbol{s}(t)-\boldsymbol{s}_{m}\right)}$

where $\boldsymbol{s}_{m}$ is the mean stress deviator, which corresponds to the co-ordinates of the center of the smallest hyper-sphere enclosing the path described by the stress deviator $\boldsymbol{s}(t)$.

Let us only remark that a shear term in both expressions assures that no microcracks are formed in the slip bands. Generalizing the observations of Winter (1974) that PSB's begin to appear when the stress amplitude reaches a limit value denoted by $k_{s}$, a crack initiation criterion is defined by Papadopoulos (1987) as:

$k^{*}=\max _{t} k(t) \leqslant k_{s}$

The $k_{s}$ limit value is larger than the yield limit $\sigma_{y}$ of the crystal $\left(k_{s}>\sigma_{y}\right)$, but smaller than the macroscopic yield limit and the endurance limit. One can note that the pressure term takes into account the local grain distribution and assure a good match with experiments but is not deduced from a precise homogenization reasoning as the shear term.

\subsection{Shakedown, dissipation and HCF}

The plasticity developed in some grains will produce an instantaneous mesoscopic plastic dissipation $d_{p}$. By using the previous framework corresponding to the Section 2.1, $d_{p}$ can be computed from:

$d_{p}=\boldsymbol{\sigma}: \dot{\boldsymbol{\varepsilon}}^{p}$

As a consequence, the Papadopoulos fatigue criterion can be redefine in terms of dissipation. A sketch of the ideas presented next is drawn on Fig. 9.

(1) If $k^{*} \leqslant \sigma_{y}$, with $\sigma_{y}$ the yield limit of the grain, no plasticity occurs at the mesoscopic scale. As a consequence a fully elastic behavior is assured and no crack initiation will occur. Moreover:

$$
k^{*} \leqslant \sigma_{y} \quad \text { if and only if } \quad d_{p}=0
$$




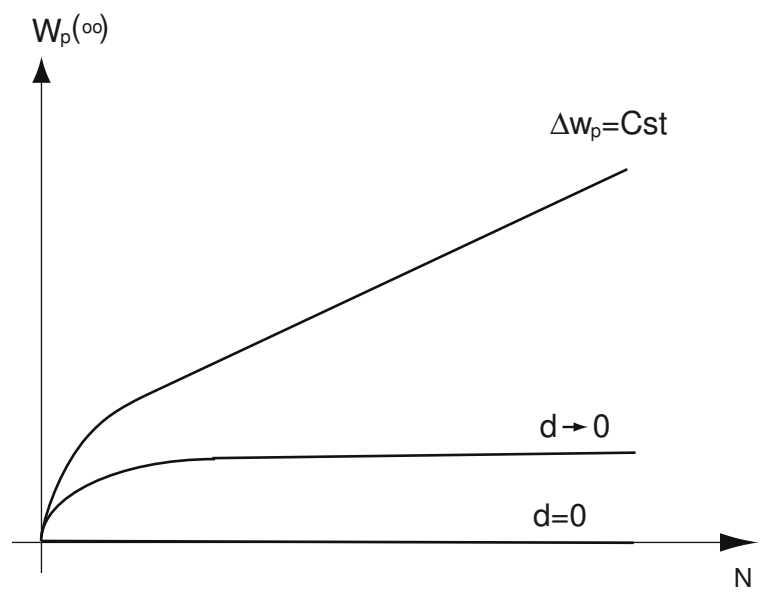

Fig. 9. Theoretical evolution of the mesoscopic cumulated mechanical dissipation $d_{\text {cumul }}$

(2) If $\sigma_{y} \leqslant k^{*} \leqslant k_{s}$, the grain plastifies but reaches an elastic shakedown state and, therefore, no crack initiation can be observed. As an elastic shakedowned state is attained, the shakedown theorem assure that the cumulated plastic work $W_{p}(\infty)$ is bounded, as demonstrated by Nguyen (2003):

$W_{p}(\infty)=\int_{0}^{\infty} \boldsymbol{\sigma}(t): \dot{\boldsymbol{\varepsilon}}^{p}(t) d t=\int_{0}^{\infty} d_{p}(t) d t<\infty$

Therefore $d_{p}$ leads to zero with the number of loading cycles and it can be stated that:

$\sigma_{y} \leqslant k^{*} \leqslant k_{s} \quad$ if and only if $\quad \lim _{t \rightarrow \infty} d_{p}(t)=0$

(3) $k_{s}<k^{*}$, elastic shakedown is not reached and a crack will initiate in one of the slip bands of the misoriented grains. The material assumptions of the fatigue criterion imply that the isotropic hardening is saturated at $k_{\mathrm{s}}$ and only kinematic hardening is activated. Moreover, applying Halphen's shakedown theorem for elastoplastic structures with kinematic hardening Halphen (1976) indicates that under a periodic loading, the structure will have a periodic solution for stress and strain tensors. As elastic shakedown is not possible anymore, the misoriented grain reaches a plastic shakedown state. Therefore, in this case, it can simply be proved that the plastic work over a loading cycle:

$\Delta w_{p}=\int_{c y c l e} d_{p} d t$

is constant. Then,

$k^{*}>k_{s} \quad$ if and only if $\quad \lim _{N \rightarrow+\infty} \Delta w_{p}=$ constant

The three types of evolution of the plastic dissipation: (4)-(6) are schematically represented on Figs. 9 and 10. The plots represent the evolution of the cumulated plastic work $W_{p}(\infty)$ versus the number of cycles of a fatigue test and the plastic work per cycle $\Delta w_{p}$ versus the applied load

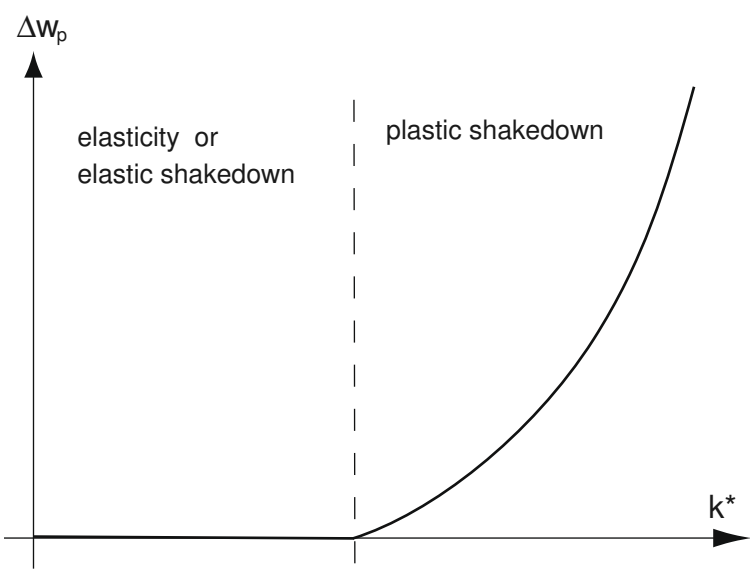

Fig. 10. Theoretical evolution of the mesoscopic dissipated energy per cycle $\Delta w$ versus the previous defined stress $k^{*}$.

represented by $k^{*}$, respectively. Fig. 10 shows that the stabilized dissipative regime depends on the loading: up to a critical load, denoted $k_{s}$ and related to the definition of shakedown in the fatigue criterion, no dissipation can be observed. As loading is increased, a plastic shakedown regime is obtained which conducts to limited endurance or low cycle fatigue.

Both Figs. 9 and 10 relate directly the fatigue regime with different plastic dissipative states. Unfortunately, direct experimental measurements of the plastic dissipation at the mesoscopic scale are not possible. However, different indirect measurement techniques can be employed; for example, measuring the plastic dissipation through the temperature increase. Therefore, the next section will present the heat Eq. (7), as deduced from the Thermodynamics of Irreversible Processes (TIP), which exhibits a coupling between temperature and plastic dissipation (see Appendix B). Different steps will be discussed and related to experimental results.

\section{A thermoplastic two scales model}

\subsection{Mesoscopic heat balance equation}

In the Dang Van and Papadopoulos HCF framework (Section 2.1), the considered REV of material is elastic and the plasticity is localized is some grains, represented then as a plastic inclusion of volume fraction $f_{v}$ in an elastic matrix. This assumption is justified as the effect of very small macroscopic plastic strains is negligible in the interaction law (A.1) and therefore, in the estimation of the macroscopic stresses. However, it will be shown that this is no more the case for the estimation of the macroscopic dissipation. Therefore, in the following, we generalize the Dang Van assumptions by considering, as a first step, a plastic matrix where the macroscopic strains correspond to the mean plastic strains in the REV, i.e. $\boldsymbol{E}^{p}=f_{v} \varepsilon^{p}$, where $\boldsymbol{E}^{p}$ and $\boldsymbol{\varepsilon}^{p}$ are respectively, the macroscopic and mesoscopic plastic strain tensors. As a consequence of the relation (A.2), $\operatorname{tr}(\boldsymbol{\sigma})=\operatorname{tr}(\boldsymbol{\Sigma})$; the mesoscopic heat balance equation can then be simplified as (see the Appendix B): 
$\rho C_{v} \dot{T}-\operatorname{div}(\boldsymbol{\kappa} \cdot \operatorname{grad}(T))=r-\alpha T \operatorname{tr} \dot{\boldsymbol{\Sigma}}-9 K \alpha^{2} T \dot{T}+\Phi$

where $\rho$ is the density, $C_{v}$ is the specific heat, $T$ is the absolute temperature, $\boldsymbol{\kappa}$ is the second rank tensor of thermal conductivity, $\alpha$ is the thermal expansion coefficient and $K$ is the bulk modulus. $\boldsymbol{E}^{e}$ and $\boldsymbol{\varepsilon}^{e}$ denote the macroscopic, respectively, mesoscopic, elastic strain tensors. In the right terms of this Eq. 7, $r$ is the distribution of external heat sources; both following terms correspond to the reversible thermoelastic coupling and $\Phi$ is the intrinsic dissipation, defined as:

$\Phi=\boldsymbol{\Sigma}: \dot{\boldsymbol{E}}^{p}+f_{v}\left[\boldsymbol{\Sigma}: \dot{\boldsymbol{E}}^{e}-\boldsymbol{\sigma}: \dot{\boldsymbol{\varepsilon}}^{e}-\frac{2}{3} h \boldsymbol{\varepsilon}^{p}: \dot{\boldsymbol{\varepsilon}}^{p}\right]$

where $h$ is the mesoscopic hardening modulus. Noting that $\boldsymbol{E}^{p}=f_{v} \boldsymbol{\varepsilon}^{p}$, the interaction law (A.1) and the relation (A.2) conducts to the following relation between $\sigma$ and $\Sigma$ :

$\boldsymbol{\Sigma}=\boldsymbol{\sigma}+2 \mu(1-b)\left(1-f_{v}\right) \boldsymbol{\varepsilon}^{p}$

In all the cases, the same elastic behavior at the mesoscopic and the macroscopic scale is assumed. Therefore, the previous relation implies:

$\boldsymbol{E}=\boldsymbol{\varepsilon}^{e}+(1-b)\left(1-f_{v}\right) \boldsymbol{\varepsilon}^{p}$

Then, introducing these both relations in the previous expression of $\Phi$, and by neglecting the $f_{v}^{2}$ and $f_{v}^{3}$ terms, the following relation is obtained:

$$
\begin{aligned}
\Phi= & f_{v} \boldsymbol{\sigma}: \dot{\boldsymbol{\varepsilon}}^{p}+f_{v}\left(2 \mu(1-b)(2-b)-\frac{2}{3} h\right) \boldsymbol{\varepsilon}^{p}: \dot{\boldsymbol{\varepsilon}}^{p} \\
& +f_{v}(1-b)\left(\boldsymbol{\sigma}: \dot{\boldsymbol{\varepsilon}}^{p}+\dot{\boldsymbol{\sigma}}: \boldsymbol{\varepsilon}^{p}\right)
\end{aligned}
$$

where $b$ is a constant depending on the homogenization scheme and $\mu$ is one of the Lamé's coefficient (see Appen$\operatorname{dix}$ A). The first term corresponds to the plastic dissipation in the REV. The other terms are associated to the mechanical power stored in the linear kinematic hardening and in the mesoscopic residual stresses due to the strain incompatibility between the inclusion and the matrix. It is important to note that, in the proposed modeling, these last terms are reversible Bornert et al., 2001b. Therefore, by considering a periodic cyclic loading, one has:

$\int_{\text {cycle }}\left[\left(2 \mu(1-b)(2-b)-\frac{2}{3} c\right) \boldsymbol{\varepsilon}^{p}: \dot{\boldsymbol{\varepsilon}}^{p}+(1-b)\left(\boldsymbol{\sigma}: \dot{\boldsymbol{\varepsilon}}^{p}+\dot{\boldsymbol{\sigma}}: \boldsymbol{\varepsilon}^{p}\right)\right] d t=0$

This simple modeling is a first step. However, one has to point out that other models consider non reversible stored energy. This is the particular case of the dislocation based model of Tanaka and Mura (1981). By considering irreversible motions in dislocation pile-ups, Tanaka and Mura (1981) proposed a ratcheting mechanisms conducting to the creation of extrusion or intrusion. This type of modeling can therefore modify the intrinsic dissipation, conducting to other cyclic evolutions. This aspect certainly has to be improved in future works.

The coupled heat Eq. 7 can now be solved in the particular case of a tension-compression experiment, defined by a macroscopic stress tensor of the form:

$\boldsymbol{\Sigma}=\Sigma_{11} \sin (\omega t) \boldsymbol{e}_{1} \otimes \boldsymbol{e}_{1}$

and, in the absence of heat sources, $r=0$.
Following Boulanger et al. (2004) or Doudard et al. (2005), it is assume that the specimen is a thin plate where the conduction phenomenon is isotropic $(\boldsymbol{\kappa}=\kappa \boldsymbol{I})$. In order to simplify the computations, their estimation of the thermal exchanges associated term (see Boulanger, 2004 for more details) is used:

$-\operatorname{div}(\boldsymbol{\kappa} \cdot \operatorname{grad}(T)) \simeq \rho C_{v} \frac{\theta}{\tau_{e q}}$

where $\tau_{e q}$ is a constant representative of the heat exchanges of the specimen with the environment through the surface in contact with the air and the grips and $\theta=T-T_{0}$. This hypothesis is acceptable provided the bulk temperature is considered equal to the surface temperature of the specimen and homogeneous along the surface, and the temperature variations are small. If the previous experimental observations of Stärk (1980) (see Fig. 5) are considered, under low level loadings, this hypothesis seems to be acceptable.

Then, the mesoscopic heat balance Eq. (7) can be simplified as:

$$
\begin{aligned}
\left(1+\frac{9 K \alpha^{2} T}{\rho C_{v}}\right) \dot{T}+\frac{\theta}{\tau_{e q}}= & \frac{f_{v}}{\rho C_{v}}\left(\boldsymbol{\sigma}: \dot{\boldsymbol{\varepsilon}}^{p}-\frac{2}{3} h \boldsymbol{\varepsilon}^{p}: \dot{\boldsymbol{\varepsilon}}\right. \\
& +2 \mu(1-b)(2-b) \boldsymbol{\varepsilon}^{p}: \dot{\boldsymbol{\varepsilon}}^{p} \\
& \left.+(1-b)\left(\boldsymbol{\sigma}: \dot{\boldsymbol{\varepsilon}}^{p}+\dot{\boldsymbol{\sigma}}: \boldsymbol{\varepsilon}^{p}\right)\right)-\frac{\alpha T}{\rho C_{v}} \operatorname{tr}(\dot{\boldsymbol{\Sigma}})
\end{aligned}
$$

This equation can easily be integrated provided the mesoscopic plastic strains are determined. They will be computed from the macroscopic loading using the homogenization scheme and a radial-return plastic integration algorithm. A simple numerical integration scheme is given in the Appendix C.

\subsection{Results and discussion for a cyclic tension-compression experiment}

It is also important to explain the cyclic technique loading used in this type of experiment, as explained in Doudard etal. (2005), Fargione et al. (2002) and Luong (1995). Different stress amplitudes are applied on the specimen and each amplitude is maintained until a stabilization of the temperature is observed. The stabilized temperature amplitude is defined as $\theta_{\text {stab }}=T_{\text {stab }}-T_{0}$, with $T_{0}$, the initial temperature. During the tests, it can be observed on the one hand, the cyclic variation of temperature, i.e. at each cycle a heating in compression and a cooling in extension as predicted by the thermoelastic coupling (see Fig. 15), and, on the other hand, a small but continuous growth of the mean temperature due to the plastic dissipation of the misoriented grains, as observed macroscopically by Dillon (1963) or Stärk (1980) (see also Figs. 3 and 4).

In order to integrate numerically the previous Eq. (8), the material parameters displayed in Tables 1 and 2 are used. They correspond in large to the ferrite-martensite dual phase steels (DP60 and DP600) as in Boulanger (2004) or Doudard (2004), Doudard et al. (2005). $\rho, \alpha, C_{v}$, $E, v, \tau_{\text {eq }}$ have been deduced from Boulanger et al. (2004), Doudard (2004) and Doudard et al. (2005). The estimation of the mesoscopic tensile yield stress $\sigma_{y}=120 \mathrm{MPa}$ is 
Table 1

Thermal material parameters used for the DP60 steel, after Doudard (2004) and Boulanger (2004).

\begin{tabular}{llll}
\hline$\rho\left(\mathrm{kg} \mathrm{m}^{-3}\right)$ & $\alpha\left(\mathrm{K}^{-1}\right)$ & $C_{v}\left(\mathrm{~J} \mathrm{~kg}^{-1} \mathrm{~K}^{-1}\right)$ & $\tau_{e q}(s)$ \\
\hline 7800 & 0.00001 & 460 & 80 \\
\hline
\end{tabular}

Table 2

Mechanical material parameters used for the DP60 steel, after Doudard (2004), Boulanger (2004) and Monchiet et al. (2006).

\begin{tabular}{llll}
\hline$E(\mathrm{MPa})$ & $v$ & $h(\mathrm{MPa})$ & $\sigma_{y}(\mathrm{MPa})$ \\
\hline 210,000 & 0.3 & 1000 & 120 \\
\hline
\end{tabular}

extracted from Monchiet et al. (2006). For the plastic hardening behavior, a hardening modulus $h=1000 \mathrm{MPa}$ has been chosen. The proposed value denotes small distance to a perfect plastic behavior, which is considered as a first approximation. Let us remark that a perfectly plastic model would not be acceptable numerically for the Sachs model as this would imply infinite plastic strains as soon as the stress is beyond the yield limit (see Eq. (C.6) with $b=1$ and $h=0$ ).

A delicate point is the estimation of the volume ratio of plastic inclusions, $f_{v}$. The present values are based on a previous work of Cugy and Galtier (2002) on low carbon steel. The only available data are the relative surface covered by slip bands, which can not exactly be correlated to the volume ratio. A first assumption is to consider this surface ratio as representative of $f_{v}$ and to take into account the values obtained for low carbon steel. The values observed by Cugy and Galtier are closed to zero near the endurance limit and less than $20 \%$ when the stress range is equal to the standard yield stress at $0.2 \%$ (see Fig. 11). As a consequence, a value of $f_{v}=3 \%$ is chosen for a stress amplitude of $180 \mathrm{MPa}$, a value of $f_{v}=10 \%$ for a stress amplitude of 250MPa and a value of $f_{v}=20 \%$ for a stress amplitude of 300MPa which correspond to the three simulated loadings. Of course, this coarse assumption has to be refined in future work. The example discussed next corresponds to tensile fatigue tests at $R_{\sigma}=-1$ with a loading frequency

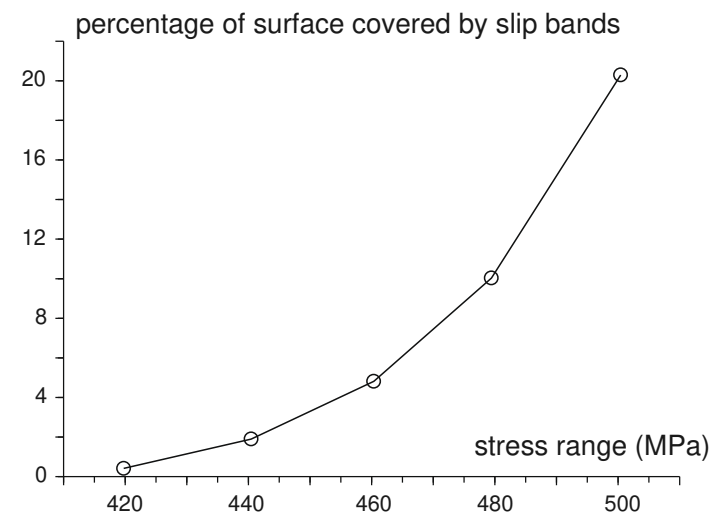

Fig. 11. Percentage of surface covered by slip bands for different stress amplitudes. The results come from a previous work of Cugy and Galtier (2002) on low carbon steel whose endurance limit $\sigma_{D}=235 \mathrm{MPa}$.

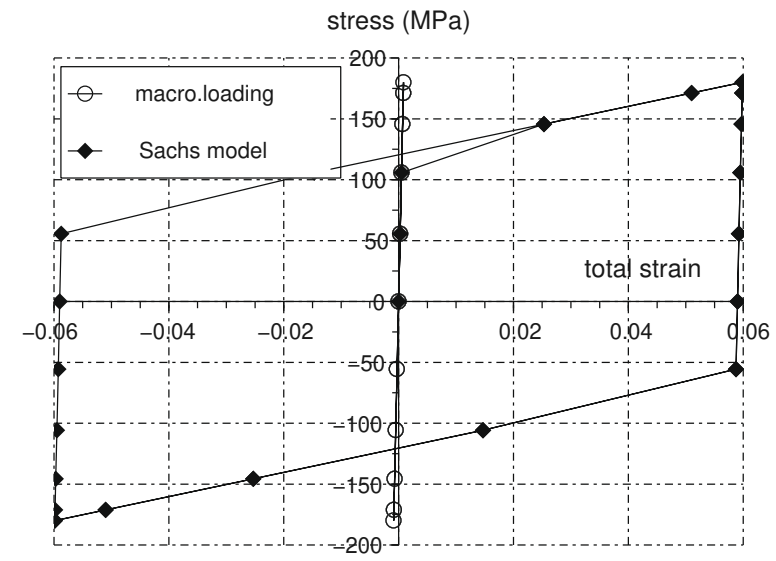

Fig. 12. Stress-strain curves corresponding to the elastic macroscopic loading and to the mesoscopic response for the Sachs scheme.

of $50 \mathrm{~Hz}$ and stress amplitudes of 180, 250 and $300 \mathrm{MPa}$, performed by Boulanger (2004). It presents the same qualitative characteristics as results obtained by Luong (1995) on a XC55 steel and Fargione et al. (2002) on different steels. First, the macroscopic and mesoscopic stress-strain curves for the different homogenization scheme are compared. In Fig. 12, the results obtained in the case of the Sachs scheme are displayed. As expected, due to the equality between macroscopic and mesoscopic stress fields and to $h \ll E$, mesoscopic plastic strains in the inclusion are very important (6\%), which is not realistic, and it can be concluded that the Sachs scheme is not appropriate for this type of loading. Fig. 13 presents the differences between Lin-Taylor's and KrÖner's schemes. It can be observed that the stress-strain curves are similar, with stresses and strains of the same order of magnitude. By analyzing the different obtained results, it can be concluded that only Taylor's and Kröner's homogenization schemes enable the achievement of consistent qualitative results, as proved by the studied example displayed on Fig. 14. The quantitative predictions are cyclic temperature amplitude of

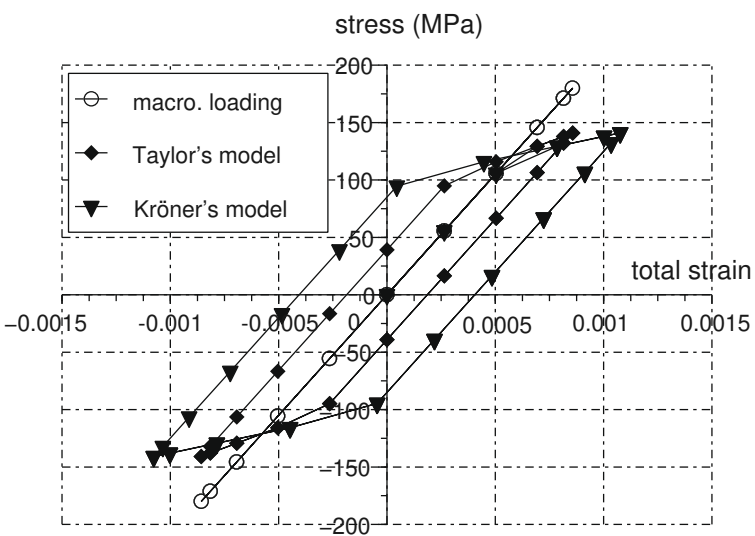

Fig. 13. Stress-strain curves corresponding to the elastic macroscopic loading and to the mesoscopic responses for the Kröner's and Taylor's schemes. 


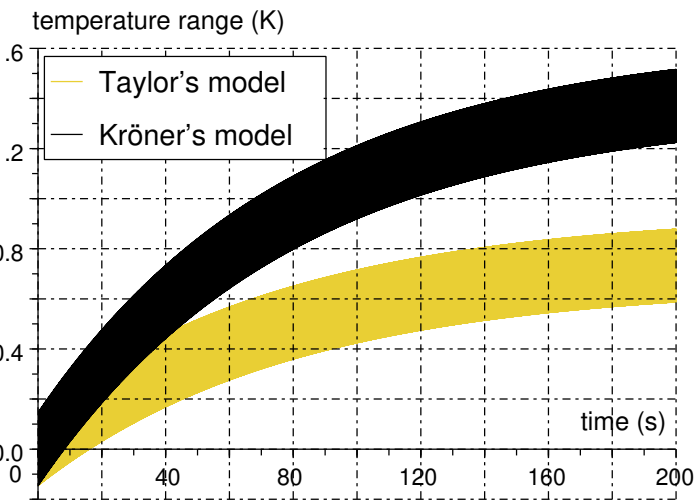

Fig. 14. Evolution of the temperature range during cyclic loading for the Taylor's and Kröner's schemes for a stress amplitude of $180 \mathrm{MPa}$.

approximately $0.3 \mathrm{~K}$ (see on Fig. 15), due to the thermoelastic coupling, and a mean temperature increase of about 0.4-0.7 $\mathrm{K}$ which is in the order of magnitude of the experimental observations of Boulanger et al., 2004 and which is due to the intrinsic dissipation.

A direct comparison of the numerical prediction of the mean temperature increase with respect to the applied load amplitude with experiments of Boulanger et al. (2004) is presented on Fig. 16. A relatively good match between predictions and experiments can be observed for low level of loadings. The quality of the match is decreasing with increasing loading but this dissipative regime corresponds to limited endurance and, progressively, to LCF. Then, the mismatch can be explained, in particular, by the simple slip system and the confined plasticity hypothesis which are very restrictive and not representative of the local behavior. Therefore, LCF has to be analyzed with other conditions which correspond to macroscopic plasticity at the REV scale. This is the matter of future works.

\section{Conclusion}

In this paper, a dissipative framework is proposed, which enables the treatment of metals fatigue, indepen-

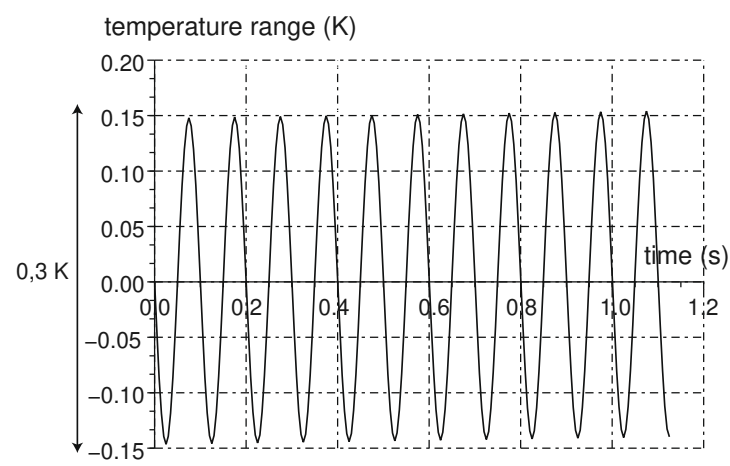

Fig. 15. Evolution of the temperature range during the first 10 cycles for the Kröner's scheme: the quasi-sinusoidal response is due to the thermoelastic coupling.

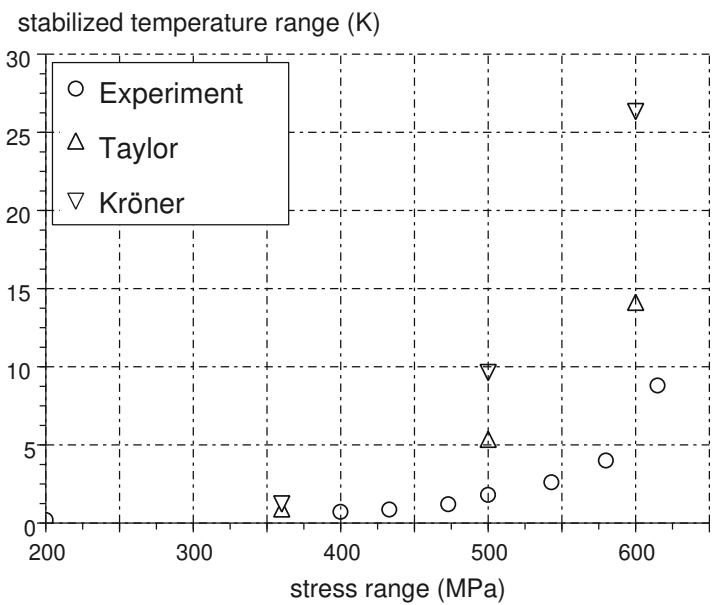

Fig. 16. Evolution of the stabilized mean temperature with respect to the loading amplitude. The experimental results stem from Boulanger (2004).

dently of the mechanical loading domain. It follows the pioneering works of Dang Van in HCF and is based on the shakedown concepts. The theoretical analysis at the mesoscopic scale, compared to existing experimental results, showed the validity of this approach. In order to propose a complete unified dissipative treatment in fatigue, three particular aspects have to be examine: the mean stress and the phase difference effects in HCF and the extension of this approach to LCF. For the first two points, the recent work of Monchiet et al. (2006, 2008), coupling plasticity and damage in HCF, seems to be promising and could also allow a re-interpretation of the existing LCF criteria based on dissipated energy, whose last contribution postulates a linear relation between dissipated energy and hydrostatic pressure (Amiable et al., 2006a,b; Park and Nelson, 2000). Therefore, the extension to LCF seems to come straightforward and will be the objective of future works.

\section{Appendix A. Meso-macro passage: Kröner's self- consistent scheme}

In the present context of a plastic inclusion in an elastoplastic matrix, in the case of confined plasticity, the relations between mesoscopic and macroscopic fields can be reached, for example, using one of the following homogenization assumptions (see for example Cano et al., 2004; Monchiet et al., 2006):

- Lin-Taylor supposes strain equality: $\boldsymbol{\varepsilon}=\boldsymbol{E}$. This is the hypothesis of the initial Dang Van or Papadopoulos fatigue criterion.

- Sachs supposes stress equality: $\boldsymbol{\sigma}=\boldsymbol{\Sigma}$

- Kröner assumes:

$$
\boldsymbol{\sigma}=\boldsymbol{\Sigma}-\mathbb{C}:(\mathbb{\square}-\mathbb{P}: \mathbb{C}):\left(\boldsymbol{\varepsilon}^{p}-\boldsymbol{E}^{p}\right)
$$

where $\mathbb{C}$ and $\mathbb{P}$ are respectively, the fourth rank elastic moduli and Hill tensors. In the particular case of an idealized spherical inclusion, $\mathbb{P}$ reads: 
$\mathbb{P}=\frac{a}{3 K} \sqrt{ }+\frac{b}{2 \mu} \mathbb{K} \quad$ with : $\quad a=\frac{3 K}{3 K+4 \mu}$ and

$b=\frac{6}{5} \frac{K+2 \mu}{3 K+4 \mu}$

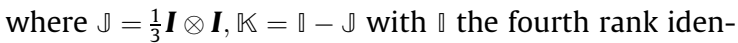
tity tensor, $K$ is the bulk modulus, $K=3 \lambda+2 \mu$ and $\lambda, \mu$ are the Lamé's parameters.

In all the cases, the same elastic behavior at the mesoscopic and the macroscopic scale is assumed. Then, the relation between mesoscopic and macroscopic fields can be written in the general form:

$\boldsymbol{\sigma}=\boldsymbol{\Sigma}-\mathbb{C}^{*}:\left(\boldsymbol{\varepsilon}^{p}-\boldsymbol{E}^{p}\right)=\boldsymbol{\Sigma}+\boldsymbol{\rho}^{*}$

where $\boldsymbol{\rho}^{*}$ should be interpreted as a mesoscopic residual stress field.

The particular cases of each model are obtained for the next form of $\mathbb{C}^{*}$ :

- for Lin-Taylor's model, $\mathbb{C}^{*}=\mathbb{C}$

- for Sachs model, $\mathbb{C}^{*}=0$ and

- for Kröner's scheme, $\mathbb{C}^{*}=\mathbb{C}:(\mathbb{\square}-\mathbb{P}: \mathbb{C})$

In the case of isotropic elastic behavior with a classically defined deviatoric plasticity, one can remark that:

$\operatorname{tr}(\boldsymbol{\sigma})=\operatorname{tr}(\boldsymbol{\Sigma}) \quad \boldsymbol{\rho}^{*}=-\mathbb{C}^{*}:\left(\boldsymbol{\varepsilon}^{p}-\boldsymbol{E}^{p}\right)=-2 \mu(1-b)\left(\boldsymbol{\varepsilon}^{p}-\boldsymbol{E}^{p}\right)$

where $b=1$ for the Sachs's model and $b=0$ for the LinTaylor's model.

\section{Appendix B. Continuum thermodynamics}

The Thermodynamics of Irreversible Processes (TIP) is described in numerous works (for example Lemaitre and Chaboche, 1985; Maugin, 1992) and enables the determination of the heat coupled equation connecting the thermal field and the mechanical fields. By defining a free energy $\Psi$ depending on state variables $\alpha_{j},(j=1,2, \ldots, n)$, this heat equation can be written as:

$\left.\rho C_{v} \dot{T}=r+\operatorname{div}(\boldsymbol{\kappa} \cdot \operatorname{grad}(T))+\boldsymbol{\Sigma}: \dot{\boldsymbol{E}}+\rho T \frac{\partial^{2} \Psi}{\partial T \partial \alpha_{j}} \dot{\alpha}_{j}-\rho \frac{\partial \Psi}{\partial \alpha_{j}} \dot{\alpha}_{j}\right)$

where $\rho$ is the density, $C$ the specific heat, $T$ the absolute temperature, $r$ the distribution of external heat sources, $\boldsymbol{k}$ the second rank tensor of thermal conductivity, $\boldsymbol{E}$ the macroscopic strain tensor, i.e. at the boundary of the REV, and $\boldsymbol{\Sigma}$ the macroscopic stress tensor.

One can remark that this expression of the heat coupled Eq. (B.1) is completely independent of the mechanical constitutive law. In the last term on the right, one find the intrinsic dissipation $\Phi$ :

$\Phi=\boldsymbol{\Sigma}: \dot{\boldsymbol{E}}-\rho \frac{\partial \Psi}{\partial \alpha_{j}} \dot{\alpha}_{j}$

which takes into account the mechanical power contribution and the stored part in the material, associated to a coupling thermomechanical term $\rho T \frac{\partial^{2} \Psi}{\partial T \partial \alpha_{j}} \dot{\alpha}_{j}$.
In this paper, a macroscopically plastic REV of matrixinclusion type is considered. The inclusion admits an elastoplastic behavior and, for sake of simplicity, a linear kinematic hardening. As the plasticity is supposed very confined in a few grains, the volume fraction of plastic inclusion, $f_{v}$, is considered low. The matrix is considered perfectly plastic and its volume fraction is $\left(1-f_{v}\right)$. The state variables are then $\boldsymbol{E}^{e}, \boldsymbol{\varepsilon}_{e}$ and $\boldsymbol{\varepsilon}_{p}$ and their thermodynamical associated forces, $\boldsymbol{\Sigma}$ and $\boldsymbol{\sigma}$ the macro- and mesoscopic stress tensors and $\boldsymbol{x}$ the stress tensor associated to the kinematic hardening. Consequently, the Helmholtz's free energy is decomposed in two parts, $\Psi_{\text {mat }}$ associated to the matrix and $\Psi_{\text {in }}$ associated to the inclusion, which conducts to:

$\Psi_{\text {in }}=\Psi_{\text {in }}\left(T, \boldsymbol{\varepsilon}^{e}, \boldsymbol{\varepsilon}^{p}\right) \quad \Psi_{m a t}=\Psi_{m a t}(T, \boldsymbol{E})$

In the case of the isotropic elasticity and thermoelasticity, both linear, the Helmholtz free energy $\Psi_{\text {mat }}$ takes the following form in the matrix:

$$
\begin{aligned}
\rho \Psi_{\text {mat }}\left(T, \boldsymbol{E}^{e}\right)= & \frac{1}{2}\left(\lambda \operatorname{tr}\left(\boldsymbol{E}^{e}\right)^{2}+2 \mu \operatorname{tr}\left(\boldsymbol{E}^{e^{2}}\right)\right) \\
& -(3 \lambda+2 \mu) \alpha \theta \operatorname{tr}\left(\boldsymbol{E}^{e}\right)+\frac{C_{v} \theta^{2}}{2 T_{0}}
\end{aligned}
$$

and, in the same way, in the plastic inclusion:

$$
\begin{aligned}
\rho \Psi_{i n}\left(T, \boldsymbol{\varepsilon}^{e}, \boldsymbol{\varepsilon}^{p}\right)= & \frac{1}{2}\left(\lambda \operatorname{tr}\left(\boldsymbol{\varepsilon}^{e}\right)^{2}+2 \mu \operatorname{tr}\left(\boldsymbol{\varepsilon}^{e^{2}}\right)\right) \\
& -(3 \lambda+2 \mu) \alpha \theta \operatorname{tr}\left(\boldsymbol{\varepsilon}^{e}\right)+\frac{1}{3} h \operatorname{tr}\left(\boldsymbol{\varepsilon}^{p^{2}}\right)+\frac{C_{v} \theta^{2}}{2 T_{0}}
\end{aligned}
$$

Then the following heat coupled equation is obtained:

$$
\begin{aligned}
\rho C_{v} \dot{T}= & r+\operatorname{div}(\boldsymbol{\kappa} \cdot \operatorname{grad}(T))+\boldsymbol{\Sigma}: \dot{\boldsymbol{E}}-\left(1-f_{v}\right) \\
& \times\left(\boldsymbol{\Sigma}: \dot{\boldsymbol{E}}^{e}+\alpha \operatorname{Ttr}(\dot{\boldsymbol{\Sigma}})+9 K \alpha^{2} T \dot{T}\right) \\
& -f_{v}\left(\boldsymbol{\sigma}: \dot{\boldsymbol{\varepsilon}}^{e}+\frac{2}{3} h \boldsymbol{\varepsilon}^{p}: \dot{\boldsymbol{\varepsilon}}^{p}+\alpha \operatorname{Ttr}(\dot{\boldsymbol{\sigma}})+9 K \alpha^{2} T \dot{T}\right)
\end{aligned}
$$

As $\operatorname{tr}(\dot{\boldsymbol{\sigma}})=\operatorname{tr}(\dot{\boldsymbol{\Sigma}})$ (see relation (A.2)), this equation can be simplified and conducts to:

$$
\begin{aligned}
& \rho C_{v} \dot{T}-\operatorname{div}(\boldsymbol{\kappa} \cdot \operatorname{grad}(T))=r-\alpha \operatorname{Ttr}(\dot{\boldsymbol{\Sigma}})+9 K \alpha^{2} T \dot{T} \\
& \quad+\boldsymbol{\Sigma}: \dot{\boldsymbol{E}}-\left(1-f_{v}\right) \boldsymbol{\Sigma}: \dot{\boldsymbol{E}}^{e}-f_{v}\left(\boldsymbol{\sigma}: \dot{\boldsymbol{\varepsilon}}^{e}+\frac{2}{3} h \boldsymbol{\varepsilon}^{p}: \dot{\boldsymbol{\varepsilon}}^{p}\right) \\
& \quad=r-\alpha \operatorname{tr}(\dot{\boldsymbol{\Sigma}})+9 K \alpha^{2} T \dot{T}+\Phi
\end{aligned}
$$

The first term on the right, $r$, corresponds to external heat sources, the two next terms, to the thermoelastic coupling and the last terms $\Phi$, to the intrinsic dissipation:

$\Phi=\boldsymbol{\Sigma}: \dot{\boldsymbol{E}}^{p}+f_{v}\left[\boldsymbol{\Sigma}: \dot{\boldsymbol{E}}^{e}-\boldsymbol{\sigma}: \dot{\boldsymbol{\varepsilon}}^{e}-\frac{2}{3} h \boldsymbol{\varepsilon}^{p}: \dot{\boldsymbol{\varepsilon}}^{p}\right]$

\section{Appendix C. Incremental determination of the mesoscopic fields}

\section{C.1. Plastic strain increment computation}

The incremental problem which is considered here consists in the determination of the increment of the mesoscopic plastic strain tensor due to an increment of the elastic macroscopic stress tensor. The framework is the 
elastoplasticity, with a linear kinematic hardening rule and the previous Self-Consistent Scheme (SCS) presented in the Appendix A. In this way, one can first define the relative stress $\sigma^{*}$ as follow:

$\boldsymbol{\sigma}^{*}=\boldsymbol{\sigma}-\frac{2}{3} h \boldsymbol{\varepsilon}^{p}$

where $h$ is the hardening modulus. This stress can be expressed as a function of the macroscopic stress tensor $\mathbf{\Sigma}$ by using the SCS interaction corresponding to the Eq. (A.1). The macroscopic plastic strain tensor $\boldsymbol{E}^{p}$ is defined as the mean of the plastic strain in the REV, i.e. $\boldsymbol{E}^{p}=f_{v} \boldsymbol{\varepsilon}^{p}$ :

$\boldsymbol{\sigma}^{*}=\boldsymbol{\Sigma}-\left(1-f_{v}\right) \mathbb{C}^{*}: \boldsymbol{\varepsilon}^{p}-\frac{2}{3} h \boldsymbol{\varepsilon}^{p}=\boldsymbol{\Sigma}-\left(\left(1-f_{v}\right) \mathbb{C}^{*}+\frac{2}{3} h \rrbracket\right): \boldsymbol{\varepsilon}^{p}$

Moreover, as a purely deviatoric von Mises plastic criterion is chosen, the deviator of this relative stress is written $\boldsymbol{A}=\operatorname{dev}\left(\boldsymbol{\sigma}^{*}\right)$, which can be expressed as:

$\boldsymbol{A}=\mathbb{K}: \boldsymbol{\sigma}^{*}=\operatorname{dev}(\boldsymbol{\Sigma})-\mathbb{K}:\left(\left(1-f_{v}\right) \mathbb{C}^{*}+\frac{2}{3} h \llbracket\right): \boldsymbol{\varepsilon}^{p}$

As $\mathbb{K}: \mathbb{C}=2 \mu \rrbracket$ and:

$\mathbb{K}: \mathbb{K}=\mathbb{K} ; \mathbb{I}: \mathbb{J}=\mathbb{\rrbracket}$ and $\mathbb{K}: \mathbb{J}=\mathbb{J}: \mathbb{K}=0$

one can easily show that:

$\mathbb{K}:\left(\left(1-f_{v}\right) \mathbb{C}^{*}+\frac{2}{3} h \rrbracket\right)=\left(2 \mu(1-b)\left(1-f_{v}\right)+\frac{2}{3} h\right) \rrbracket$

Therefore, at the increment $n$ :

$\boldsymbol{A}_{n}=\operatorname{dev}\left(\boldsymbol{\Sigma}_{n}\right)-\left(2 \mu(1-b)\left(1-f_{v}\right)+\frac{2}{3} h\right) \boldsymbol{\varepsilon}_{n}^{p}$

The objective is the determination of the relative stress at the $n+1$ increment, $\boldsymbol{A}_{n+1}$ :

$\boldsymbol{A}_{n+1}=\boldsymbol{A}_{n}+\operatorname{dev}(\Delta \boldsymbol{\Sigma})-\left(2 \mu(1-b)\left(1-f_{v}\right)+\frac{2}{3} h\right) \Delta \boldsymbol{\varepsilon}^{p}$

To determine the plastic increment, one can finally define a trial stress $\boldsymbol{A}_{n+1}^{*}$ which corresponds to a freezing plastic flow (i.e. $\Delta \boldsymbol{\varepsilon}^{p}=\mathbf{0}$ ):

$$
\begin{aligned}
\boldsymbol{A}_{n+1}^{*} & =\boldsymbol{A}_{n}+\operatorname{dev}(\Delta \boldsymbol{\Sigma}) \\
& =\operatorname{dev}\left(\boldsymbol{\Sigma}_{n}\right)-\left(2 \mu(1-b)\left(1-f_{v}\right)+\frac{2}{3} h\right) \boldsymbol{\varepsilon}_{n}^{p}+\operatorname{dev}(\Delta \boldsymbol{\Sigma})
\end{aligned}
$$

The relative stress at the increment $n+1$ is then written as:

$\boldsymbol{A}_{n+1}=\boldsymbol{A}_{n+1}^{*}-\left(2 \mu(1-b)\left(1-f_{v}\right)+\frac{2}{3} h\right) \Delta \boldsymbol{\varepsilon}^{p}$

The implicit normality rule implies the following relation at the increment $n+1$ :

$\dot{\boldsymbol{\varepsilon}}_{n+1}^{\boldsymbol{p}}=\gamma_{p} \frac{\boldsymbol{A}_{n+1}}{\left\|\boldsymbol{A}_{n+1}\right\|}=\gamma_{p} \frac{\boldsymbol{A}_{n+1}^{*}}{\left\|\boldsymbol{A}_{n+1}^{*}\right\|}=\frac{\Delta \boldsymbol{\varepsilon}^{\boldsymbol{p}}}{\Delta t}$

By using the relation (C.2), one can find:

$$
\boldsymbol{A}_{n+1}=\boldsymbol{A}_{n+1}^{*}-\left(2 \mu(1-b)\left(1-f_{v}\right)+\frac{2}{3} h\right) \Delta t \gamma \frac{\boldsymbol{A}_{n+1}^{*}}{\left\|\boldsymbol{A}_{n+1}^{*}\right\|}
$$

which is equivalent to:

$$
\left\|\boldsymbol{A}_{n+1}\right\|=\left\|\boldsymbol{A}_{n+1}^{*}\right\|-\left(2 \mu(1-b)\left(1-f_{v}\right)+\frac{2}{3} h\right) \Delta t \gamma
$$

The consistency condition (Kuhn-Tucker) $f_{n+1}=0$ corresponds to this equation:

$J_{2}\left(\boldsymbol{A}_{n+1}\right)=\sigma_{y}$

which is equivalent, by introducing the relation (C.4), to:

$\sqrt{\frac{3}{2}}\left(\left\|\boldsymbol{A}_{n+1}^{*}\right\|-\left(2 \mu(1-b)\left(1-f_{v}\right)+\frac{2}{3} h\right) \Delta t \gamma_{p}\right)=\sigma_{y}$

One can therefore obtain the plastic multiplier $\gamma$ :

$\gamma_{p}=\frac{\sqrt{\frac{3}{2}}\left\|\boldsymbol{A}_{n+1}^{*}\right\|-\sigma_{y}}{\sqrt{\frac{3}{2}}\left(2 \mu(1-b)\left(1-f_{v}\right)+\frac{2}{3} h\right) \Delta t}$

and the plastic strain increment comes straightforward with the relation (C.2):

$$
\begin{aligned}
\Delta \boldsymbol{\varepsilon}^{p} & =\gamma_{p} \Delta t \frac{\boldsymbol{A}_{n+1}^{*}}{\left\|\boldsymbol{A}_{n+1}^{*}\right\|} \\
& =\frac{\sqrt{\frac{3}{2}}\left\|\boldsymbol{A}_{n+1}^{*}\right\|-\sigma_{y}}{\sqrt{\frac{3}{2}}\left(2 \mu(1-b)\left(1-f_{v}\right)+\frac{2}{3} h\right)} \frac{\boldsymbol{A}_{n+1}^{*}}{\left\|\boldsymbol{A}_{n+1}^{*}\right\|}
\end{aligned}
$$

with $\boldsymbol{A}_{n+1}^{*}=\operatorname{dev}\left(\boldsymbol{\Sigma}_{n}\right)-\left(2 \mu(1-b)\left(1-f_{v}\right)+\frac{2}{3} h\right) \boldsymbol{\varepsilon}_{n}^{p}+\operatorname{dev}(\Delta \Sigma)$. The determination of the temperature increment $\Delta T$ is detailed in the part C. 2 of the present appendix.

\section{C.2. Integration of the heat balance equation}

The heat balance Eq. (B.2) has been simplified to the following form (8):

$$
\begin{aligned}
\left(1+\frac{9 K \alpha^{2} T}{\rho C_{v}}\right) \dot{T}+\frac{\theta}{\tau_{e q}}= & \frac{f_{v}}{\rho C_{v}}\left(\boldsymbol{\sigma}: \dot{\boldsymbol{\varepsilon}}{ }^{p}-\frac{2}{3} h \boldsymbol{\varepsilon}^{p}: \dot{\boldsymbol{\varepsilon}^{p}}\right. \\
& +2 \mu(1-b)(2-b) \boldsymbol{\varepsilon}^{p}: \dot{\boldsymbol{\varepsilon}}^{p} \\
& \left.+(1-b)\left(\boldsymbol{\sigma}: \dot{\boldsymbol{\varepsilon}}^{p}+\dot{\boldsymbol{\sigma}}: \boldsymbol{\varepsilon}^{p}\right)\right)-\frac{\alpha T}{\rho C_{v}} \operatorname{tr}(\dot{\boldsymbol{\Sigma}})
\end{aligned}
$$

For common metallic materials, the following simplification can be done:

$\frac{9 K \alpha^{2} T}{\rho C_{v}}<<1$

The heat coupled equation can then be written as:

$$
\begin{aligned}
\dot{T}+\frac{T-T_{0}}{\tau_{e q}}= & \frac{f_{v}}{\rho C_{v}}\left(\boldsymbol{\sigma}: \dot{\boldsymbol{\varepsilon}}^{p}-\frac{2}{3} h \boldsymbol{\varepsilon}^{p}: \dot{\boldsymbol{\varepsilon}}^{p}+2 \mu(1-b)(2-b) \boldsymbol{\varepsilon}^{p}: \dot{\boldsymbol{\varepsilon}}^{p}\right. \\
& \left.+(1-b)\left(\boldsymbol{\sigma}: \dot{\boldsymbol{\varepsilon}}^{p}+\dot{\boldsymbol{\sigma}}: \boldsymbol{\varepsilon}^{p}\right)\right)-\frac{\alpha T}{\rho C_{v}} \operatorname{tr}(\dot{\boldsymbol{\Sigma}})
\end{aligned}
$$

which allows to assess the temperature at the next time increment by using an explicit time integration scheme:

$$
\begin{aligned}
T_{n+1}= & \frac{\frac{T_{n}}{\Delta t}+\frac{T_{0}}{\tau_{e q}}+\frac{f_{v}}{\rho C_{v} \Delta t}\left(\boldsymbol{\sigma}_{n}: \Delta \boldsymbol{\varepsilon}^{p}-\frac{2}{3} h \boldsymbol{\varepsilon}_{n}^{p}: \Delta \boldsymbol{\varepsilon}^{p}-2 \mu(1-b)(2-b) \boldsymbol{\varepsilon}_{n}^{p}: \Delta \boldsymbol{\varepsilon}^{p}\right)}{\frac{1}{\Delta t}+\frac{1}{\tau_{e q}}+\frac{\alpha}{\rho C_{v} \Delta t} \operatorname{tr}(\Delta \boldsymbol{\Sigma})} \\
& +\frac{\frac{f_{v}}{\rho C_{v} \Delta t}\left((1-b)\left(\boldsymbol{\sigma}_{n}: \Delta \boldsymbol{\varepsilon}^{p}+\boldsymbol{\varepsilon}_{n}^{p}: \Delta \boldsymbol{\sigma}\right)\right)}{\frac{1}{\Delta t}+\frac{1}{\tau_{e q}}+\frac{\alpha}{\rho C_{v} \Delta t} \operatorname{tr}(\Delta \boldsymbol{\Sigma})}
\end{aligned}
$$




\section{References}

Amiable, S., Chapuliot, S., Constantinescu, A., Fissolo, A., 2006a. A comparison of lifetime prediction methods for a thermal fatigue experiment. Int. J. Fat. 28 (7), 692-706.

Amiable, S., Chapuliot, S., Constantinescu, A., Fissolo, A., 2006b. A computational lifetime prediction of a thermal shock experiment. Part II: Discussion on different fatigue criteria. Fat. Frac. Engng. Mat. Struct. 29 (3), 219-227.

Bornert, M., Bretheau, T., Gilormini, P., 2001a. Homogénéisation en mécanique des matériaux, Tome 1 , matériaux aléatoires élastiques et milieux périodiques. Hermès Sciences.

Bornert, M., Bretheau, T., Gilormini, P., 2001b. Homogénéisation en mécanique des matériaux, Tome 2, Comportements non linéaires et problèmes ouverts. Hermès Sciences.

Boulanger, T., 2004. Analyse par thermographie infrarouge des sources de chaleur induites par la fatigue des aciers. Ph.d Thesis, spécialité: mécanique, Université de Montpellier II, France.

Boulanger, T., Chrysochoos, A., Mabru, C., Galtier, A., 2004. Calorimetric analysis of dissipative and thermoelastic effects associated with the fatigue behavior of steels. Int. J. Fat. 26, 221-229.

Cano, F., Constantinescu, A., Maïtournam, H., 2004. Critère de fatigue polycyclique pour des matériaux anisotropes: application aux monocristaux. C.R. Mécanique 332 (2), 115-121.

Chrysochoos, A., 1985. Bilan énergétique en élastoplasticité grandes déformations. J. Méca. Th. A 4 (5), 589-614.

Coffin, L.F., 1953. A study of the effects of cyclic thermal stresses on a ductile material. Trans. ASME, 931-950.

Crossland, B., 1956. Effects of large hydrostatic pressures on tortionnal fatigue strenght of an alloy steel. In: ASME (Ed.), Proc. Int. Conf Fatigue of Metals. Institution of Mechanical Engineers, pp. 138-149.

Cugy, P., Galtier, A., 2002. Microplasticity and temperature increase in low carbon steels. In: Blom, A.F. (Ed.), Proc. 8th Int. Fatigue Congress - 3-7 June 2002. EMAS, Barnsley, pp. 549-556.

Dang Van, K., 1973. Sur la résistance à la fatigue des métaux. Sciences Technique Armement 47 (3).

Dillon, O.W., 1962a. An experimental study of the heat generated during torsional oscillations. J. Mech. Phys. Solids 10, 235-244.

Dillon, O.W., 1962b. A nonlinear thermoelasticity theory. J. Mech. Phys. Solids $10,123-131$.

Dillon, O.W., 1963. Coupled thermoplasticity. J. Mech. Phys. Solids 11, 21 33.

Dillon, O.W., 1966. The heat generated during the torsional oscillations of copper tubes. Int. J. Solids Structures 2, 181-204.

Doudard, C., 2004. Détermination rapide des propriétés en fatigue à grand nombre de cycles à partir d'essais d'échauffement. Ph.d Thesis, spécialité: Mécanique, Ecole Normale Supérieure de Cachan, France.

Doudard, C., Calloch, S., Cugy, P., Galtier, A., Hild, F., 2005. A probabilistic two-scale model for high-cycle fatigue life predictions. Fatigue Fract. Engng. Mater. Struct. 28, 279-288.

Fargione, G., Geraci, A., La Rosa, G., Risitano, A., 2002. Rapid determination of the fatigue curve by the thermographic method. Int. J. Fatigue 24 11-19.

Farren, W.S., Taylor, G.I., 1925. The heat developped during plastic extension of metals. Proc. Royal Soc. A 107, 422-451.

Gough, H., Pollard, H., 1935. The strength of metals under combined alternating stress. Proc. Inst. Mech. Eng. 131, 3-18.

Halford, G.R., 1966. The energy required for fatigue. J. Mater. 1 (1), 3-18.

Halphen, B., 1976. L'accommodation des structures élastoplastiques à écrouissage cinématique. C.R. Ac. des Sciences-série A 283 (10), 799802 .

Jiang, L., Brooks, C.R., Liaw, P.K., Wang, H., Rawn, C.J., Klarstrom, D.L., 2001a. High frequency metal fatigue: the high-cycle fatigue behavior of ULTIMET alloy. Mat. Sci. Eng. A 314, 162-175.

Jiang, L., Wang, H., Liaw, P.K., Brooks, C.R., Klarstrom, D.L., 2001b. Characterization of the temperature evolution during high-cycle fatigue of the ULTIMET superalloy; experimental and theoretical modeling. Met. Mat. Trans. A 32A, 2279-2296.

Jiang, L., Wang, H., Liaw, P.K., Brooks, C.R., Klarstrom, D.L., 2004. Temperature evolution during low-cycle fatigue of ULTIMET alloy: experiment and modeling. Mech. Mat. 36, 73-84.
Korsunsky, A.M., James, K.E., Daymond, M.R., 2004. Intergranular stresses in polycrystalline fatigue: diffraction measurement and selfconsistent modelling. Eng. Frac. Mech. 71, 805-812.

Krapez, J.C., Pacou, D., 2002. Thermography detection of early thermal effects during fatigue tests of steel and aluminum samples. In: AIP Conference Proceedings, vol. 615. pp. 1545-1552.

Lemaitre, J., Chaboche, J.L., 1985. Mécanique des matériaux solides. Dunod, Paris.

Lin, T.H., 1957. Analysis of elastic and plastic strains of a FCC crystal. J. Mech. Phys. Solids 5, 143.

Lukas, P., Kunz, L., 2001. Cyclic slip localisation and fatigue crack initiation in fcc single cristals. Material Science and Engineering A 314, 75-80.

Lukas, P., Kunz, L., 2002. Specific features of high-cycle and ultra-highcycle fatigue. Fat. Frac. Engng. Mat. Struct. 25, 747-763.

Lukas, P., Kunz, L., 2004. Role of persistent slip bands in fatigue. Phil. Mag. 84 (3-5), 317-330.

Luong, M.P., 1995. Infrared thermographic scanning of fatigue in metals. Nucl. Eng. Design 158, 363-376.

Luong, M.P., 1998. Fatigue limit evaluation of metals using an infrared thermographic technique. Mech. Mat. 28, 155-163.

Magnin, T., Driver, J., Lepinoux, J., Kubin, L.P., 1984a. Aspects microstructuraux de la déformation cyclique dans les métaux et alliages C.C. et C.F.C. - I.: consolidation cyclique. Rev. Phys. Appl. 19, 467-482.

Magnin, T., Driver, J., Lepinoux, J., Kubin, L.P., 1984b. Aspects microstructuraux de la déformation cyclique dans les métaux et alliages C.C. et C.F.C. - II.: saturation cyclique et localisation de la déformation. Rev. Phys. Appl. 19, 467-482.

Mandel, J., Zarka, J., Halphen, B., 1977. Adaptation d'une structure élastoplastique à écrouissage cinématique. Mech. Res. Commun. 4 (5).

Manson, S.S., 1953. Behaviour of materials under conditions of thermal stresses. Tech. Rep. TN 2933, N.A.C.A.

Maugin, G.A., 1992. The Thermomechanics of Plasticity and Fracture. Cambridge University Press, Cambridge.

Monchiet, V., Charkaluk, E., Kondo, D., 2006. A plasticity-damage based micromechanical modelling in high cycle fatigue. C.R. Mécanique 334 (2), 129-136.

Monchiet, V., Charkaluk, E., Kondo, D., 2008. A micromechanical explanation of the mean stress effect in High Cycle Fatigue (HCF). Mech. Res. Com. 35, 383-391.

Mughrabi, H., 1983. Fatigue crack initiation by cyclic slip irreversibilities in high cycle fatigue. In: Fatigue Mechanisms: Advances in Quantitative Measurement of Physical Damage - ASTM STP 811, pp. 5-45.

Nguyen, Q., 2003. On shakedown analysis in hardening plasticity. J. Mech. Phys. Solids (51), 101-125.

Orowan, E., 1939. Theory of the fatigue of metals. Proc. Royal Soc. 171, 79-106.

Papadopoulos, I.V., 1987. Fatigue polycyclique des métaux: une nouvelle approche. Ph.d Thesis, spécialité: Mécanique, Ecole des Ponts et Chaussées, France.

Park, J., Nelson, D., 2000. Evaluation of an energy-based approach and a critical plane approach for predicting constant amplitude multiaxial fatigue life. Int. J. Fat. 22, 23-39.

Sauzay, M., 2006. Effet de l'anisotropie élastique cristalline sur la distribution des facteurs de Schmid à la surface des polycristaux. C.R. Méca 334 (6), 353-361.

Sines, G., Waisman, J., 1959. Metal Fatigue. McGraw Hill, Berlin.

Smith, K.N., Watson, P., Topper, T.H., 1970. A stress-strain function for the fatigue of metals. J. Mater. 5 (4), 767-778.

Stärk, K.F., 1980. Thermometrische Untersuchungen zum zyklischen Verformungsverhalten metallischer Werkstoffe. Ph.d Thesis, Universität Stuttgart, Deutschland.

Suresh, S., 1998. Fatigue of materials, second edition. Cambridge University Press, Cambridge.

Tanaka, K., Mura, T., 1981. A dislocation model for fatigue crack initiation. J. Appl. Mech. 48, 97-103.

Taylor, G.I., 1938. Plastic strains in metals. J. Inst. Metals 62, 307.

Winter, A.T., 1974. A model for the fatigue of copper at low plastic strain amplitude. Phil. Mag. 30 (4), 719-738. 